\title{
Simulación de
}

\section{iluminación natural en} oficinas; implicaciones para la eficiencia visual

\author{
Simulation of natural lighting in offices; \\ implications for visual efficiency
}

Mtra. Arq. Perla Liliana Zambrano Prado Dra. Lilia Rosalía Prado León

Centro de Investigaciones en

Ergonomía, Ergonomía de

Diseño

Universidad de

Guadalajara, México

Recibido: 19 Sept. 2015 Aceptado: 4 Diciembre 2015

\section{Resumen}

I desarrollo de 36 simulaciones virtuales de iluminación natural exterioriza los aportes de luz natural en un espacio de oficina y describe sus resultados según las normas para la eficiencia visual, a través del uso de un software especializado. Se compararon tres estaciones del año, en dos horarios distintos y en dos orientaciones en donde se colocó una única ventana. El software Radiance y Autodesk Ecotect mostraron que los niveles de iluminación promedio llegan a superar hasta 27 veces los valores establecidos por Norma Oficial Mexicana en la orientación Sur, en invierno, a las 12H00. Se demuestra también que el coeficiente de reflexión del color juega un papel imprescindible, aumentando hasta en un 63\% el nivel de iluminación.

Palabras clave: color, estación del año, iluminación natural, orientación simulación.

Abstract:

This article focuses on the carrying out of 36 virtual natural lighting simulations, with the purpose to provide an overview of the contributions of the simulation of natural lighting in an office space and describe their results in the light of the rules for visual efficiency, considering seasonal variations, orientation façade, time and color through the use of specialized software. Three seasons were compared at two different times and two orientations (North and South) where only one window was placed. We used Radiance and Autodesk Ecotec software. Our results showed that during winter and facing South the average lighting levels exceed 27 times the values established by the Official Mexican Norm (NOM-025-STPS-1999 Lighting conditions in work spaces) in winter at South orientation at 12:00 hrs. It was also proven that color reflection coefficient plays an essential role in lighting, increasing up to $63 \%$ the level of illumination.

Keywords: color, natural lighting, orientation, season, simulation.. 


\section{Introducción}

Desde la antigüedad el hombre buscó la manera de llevar la iluminación natural a los espacios que habitaba. La primera fuente de luz artificial fue el fuego que en la noche o en espacios completamente oscuros como cuevas, llegó para iluminar las estancias. En el último siglo, la vida de una gran mayoría de la población se desarrolla en un mundo lleno de edificaciones; además, los espacios destinados a oficina, en los últimos cincuenta años, se han convertido en el lugar en donde cada vez un mayor número de personas pasan su vida laboral (Boyce, 2003).

Uno de los representantes más importantes de movimiento moderno es el arquitecto finlandés Alvar Aalto, una de sus obras más significativas es la Biblioteca de Viipuri que supuso un cambio en la concepción espacial arquitectónica. El estudio de las condiciones ambientales y la iluminación entre otras cosas fueron novedosas en su época.

"La iluminación de las zonas de lectura se efectúa mediante unas claraboyas cónicas que tamizan la luz, con lo que los lectores no se deslumbran ni están condicionados por cambios de luz bruscos" (Kliczkowski, 2002). Aalto estudió la iluminación y consiguió con su proyecto una sala de lectura con iluminación natural difusa que no generara sombras al lector; para él, lo más importante era el usuario y la tarea de leer cómodamente.

La luz es un fenómeno del cual el hombre tiene constantemente deseo. Como en otros sectores, se ha alcanzado una encomiable perfección cuando se trata de enfrentarse racionalmente a los problemas puramente técnicos, a los que presenta el aparataje, sus partes móviles, sus modos de producción, etc., pero la tarea principal - la de iluminar al servicio del hombre, adaptar la claridad a la higiene de sus ojos, asegurar a hombre la mejor calidad de luz -, ha sido en buena medida descuidada (Aalto, 1988; citado por Fuentes Romero, 2000).

Si bien este referente de la arquitectura, con una calidad espacial excepcional debido, entre otras cosas, a un importante estudio lumínico durante el proceso de diseño no es una oficina, sí se realizan algunas tareas similares como la lectura y la escritura; es evidente sin embargo que las soluciones arquitectónicas implementadas por Aalto podrían no funcionar en latitudes cercanas a los trópicos, pues las condiciones climáticas así como la trayectoria solar es completamente distinta.

Iluminación Natural: eficiencia y confort visual en el trabajo de oficina

La iluminación es uno de los principales factores ambientales en el área de trabajo, desde el punto de vista ergonómico. Cerca del $80 \%$ de la información del trabajo se recolecta o recibe por los ojos; el diseño de la iluminación debe proporcionar un ambiente en el cual las personas, a través del sentido de la visión puedan trabajar y desarrollarse con seguridad, eficiencia y de manera confortable (Wolska, 2011).

Un equipo de investigación sostiene que es sobre todo la presencia de la ventana y la penetración de la luz solar en el lugar de trabajo las que conducen a una mayor satisfacción de los trabajadores (Kaplan y Dana, 2001). "No hay duda de que las personas prefieren la iluminación natural por encima de la iluminación artificial como recurso primario de iluminación" (Boyce, Hunter y Howlett, 2003: 26).

El avance de la tecnología ha propiciado que cada vez sea más común el uso del computador en la oficina. De acuerdo con Prado León, Ávila Chaurand, Aceves González, González Muñoz (2009) el síndrome de la visión de computadora es uno de los padecimientos más comunes entre la gente que trabaja con monitores (American Industrial Hygiene Association, 1994b). Es un problema serio, pues existen datos que afirman que alrededor de tres cuartas partes de los usuarios de computadora lo han padecido (Singh y Wadhwa, 2006).

Entre los principales síntomas que aquejan a los trabajadores se encuentran la fatiga visual, tensión, ardor e irritación de ojos, visión borrosa o doble, dolor de cabeza; una iluminación deficiente puede producir también otros efectos negativos como mareos, sentimientos negativos y tristeza (Nussbaumer, 2014).

Esto puede entenderse si se considera que un operador de computadora realiza, en cada jornada de trabajo, entre 12 mil y 33 mil movimientos de cabeza y ojos así como entre 4 mil y 17 mil reacciones de la pupila (Alonso, Terra y Menéndez, 1995).

Frente a estos problemas causados por una iluminación inadecuada, los factores que un diseño de iluminación integral debe considerar para lograr la eficiencia visual son (Wolska, 2011; Boyce, 2011):

- La fluctuación

- $\quad$ El deslumbramiento

- $\quad$ El nivel de iluminación

Por otro lado, el color es fundamental en el nivel de iluminación porque es un factor importante para la reflexión de la luz. El presente trabajo se enfocó en el nivel de la iluminación natural, considerando variaciones de reflexión del color en techos y muros.

La iluminación interior debe cumplir tres funciones: -ISO 8995, 1989, prEN 12464 1996; citado por Wolska, 2011-:

- Asegurar la máxima seguridad -hacer cualquier peligro visible-.

- Mantener un nivel apropiado de desempeño visua -los trabajadores deben ser capaces de desempeñar sus tareas visuales, aún bajo circunstancias difíciles y durante periodos extensos-

- Proporcionar confort visual. 
El diseño de una buena iluminación siempre implica considerar el desempeño visual y del confort; estos dos elementos se encuentran íntimamente relacionados: la iluminación que es inadecuada para el desempeño de una tarea conducirá a la incomodidad visual (Boyce, 2011). En base a esto, es posible suponer que si el nivel de iluminación es adecuado para la eficiencia visual del trabajo en oficinas, entonces también implicará cierta base para el confort visual.

\section{Factores que inciden en el ambiente lumínico}

Si bien los beneficios que se obtienen al iluminar un espacio con luz natural son muy diversos, es necesario cuantificar y entender los aspectos del entorno arquitectónico y no arquitectónico como la estación del año y la hora entre otros factores que influyen en el ambiente lumínico parar, de esta manera, implementar las estrategias necesarias en el diseño del espacio a fin de cumplir con los niveles indicados por las normas; de esto se deriva el desarrollo de actividades en una forma cómoda y segura.

Es importante señalar que cada región dispone de distintos niveles de iluminación; por ello, es de relevancia contar con información del sitio en donde se desarrolle el proyecto. En este sentido, las estrategias a utilizar para crear un ambiente lumínico adecuado serán diferentes según la localización de proyecto. Por tanto, para lograr la calidad espacial en relación al ambiente lumínico es necesario conocer aspectos importantes como las principales limitaciones de la luz natural: la duración del día o la cantidad de horas de luz solar; este factor dependerá de la latitud en donde se encuentre el proyecto así como la estación del año, la iluminancia horizontal exterior, la orientación del vano por donde ingresa la luz y por último las obstrucciones externas (Yáñez, 2008).

El nivel de iluminación natural que ingresa a un espacio está constituido por tres factores externos: la luz directa que proviene del sol, la luz difusa derivada del cielo y la luz de las reflexiones de elementos externos del edificio como pueden ser otras edificaciones, los pavimentos así como la vegetación.
Por otro lado, los factores internos que afectan los niveles lumínicos del espacio corresponden al tamaño y posición de la ventana, la profundidad y la forma de la habitación y los colores de las superficies interiores (Li, Wong, Tsang y Cheung, 2006). "La luz y el color son elementos que no se pueden separar..." (Ortiz, 2004:116).

Tanto la iluminación natural como el color son elementos imprescindibles en los espacios interiores; el color brinda valores culturales, suscita emociones y crea efectos psicológicos. Aguirre y Cuvelier (2014) indican que el color en espacios interiores no puede seleccionarse considerando únicamente el aspecto estético, pues debe pensarse en la funcionalidad del espacio y la influencia del contexto.

Uno de los objetivos en espacios laborales es la productividad; en este sentido, una mala elección del color puede ayudar a aumentarla o a disminuirla o inclusive crear dificultadas a nivel visual (Ortiz, 2004).

El presente trabajo consideró el color, entre otros factores, con el fin de comparar los niveles lumínicos a través de modificar el coeficiente de reflexión, como una manera de saber cuánta luz refleja una superficie según su color; esto es el LRV (Light Reflectance Value) por sus siglas en inglés. Este es un valor que mide en porcentaje la cantidad de luz que refleja una superficie; la medición se realiza en una escala del $1-100 \%$.

La primera Figura representa dicha escala, en donde el $0 \%$ es un negro absoluto que absorbe toda la luz que incide y, por tanto, no reflejará nada mientras el 100\% representa un color blanco puro que significa que reflejará toda la luz que incida en la superficie. Elegir un color con un alto LRV podría disminuir las necesidades de la iluminación artificial; por tanto, potenciaría el recurso de la iluminación natural.

$0 \%$

$100 \%$

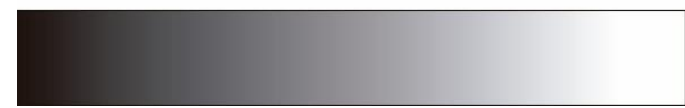

Figura 1: Ejemplo de escala del valor de reflexión de la luz del $0-100 \%$.

RV $10 \%$

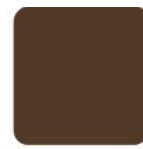

LRV $10 \%$

RGB: 83-51-30

RGB: $60-61-62$

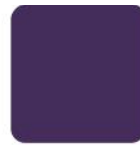

RGB: $57-45-11$

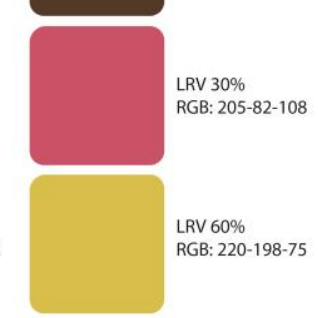

LRV $60 \%$

GB: 220-198-75

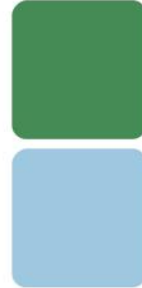

RV $30 \%$

RGB: $50-151-96$

RGB: 124-129-124

LRV $90 \%$

RGB: $243-143-237$

LRV $80 \%$

RGB: $194-230-236$

LRV $80 \%$

RGB: $245-232-226$

Figura 2: Colores con su correspondiente valor de reflexión de la luz y valores RGB, realizada a partir de la información de una web de pinturas. 
Como ejemplo, en la Figura 2, se muestran diversos colores que podrían ser aplicados a superficies interiores, con su correspondiente LRV así como se establece el valor RGB al que se ha dividido en tres: neutrales, fríos y cálidos; la información ha sido obtenida de una tienda de pinturas (Resene. The paint the professionals use, 2015).

Las últimas décadas han sido testigos de múltiples avances sobre cómo analizar numéricamente el desempeño general de la iluminación natural en los espacios interiores. Éstos incluyen una tendencia a la utilización de las simulaciones basadas en la iluminación natural; los beneficios que pueden obtenerse son el confort visual, el térmico, disminución de deslumbramiento y reducción del consumo de energía (Reinhart y Wienold, 2011).

Debido a los avances de la tecnología informática, se ha dado un aumento constante en el uso de la computadora como herramienta de simulación para evaluar los sistemas de iluminación natural. Reinhart y Wienold (2011) consideran que tanto arquitectos como autoridades deben convencerse de que los análisis de simulación de la luz de día, basado en computadoras, pueden facilitar el diseño de edificios con iluminación natural.

Radiance es un programa de simulación de iluminación que ha sido utilizada por varios investigadores para calcular iluminancias interiores (Ward y Shakespeare, 1998 citado por Li y Tsang, 2005). Se ha reportado que este software de simulación puede proporcionar una reproducción más cercana de medidas reales en edificios, en comparación con otros software de iluminación natural (Gugliermetti, Grignaffini y Bisegna, 2001 citado por Li, Cheung, Cheung y Lam, 2010).

Dentro de los parámetros más importantes a considera cuando se realiza una simulación de iluminación natural a más de la elección del software, es el modelo de cielo a utilizar; las simulaciones digitales del cielo son uno de los factores más importantes en los cálculos de iluminación natural (Kensek y Suk, 2011).

Uno de los programas que ofrecen la posibilidad de realizar simulaciones lumínicas es Ecotect Analysis. Este software dispone únicamente de dos modelos de cielo: el cubierto y el uniforme; sin embargo, al utilizar el software Desktop Radiance V2 a través de Ecotec es posible contar con una variedad más amplia de modelos de cielo.

En la actualidad los modelos de cielo más utilizados en la realización de simulaciones virtuales son los del CIE (Commission Internationale de l'eclairage) para cielo cubierto y despejado. La ubicación del proyecto es uno de los factores determinantes para elegir el modelo de cielo a utilizar en las simulaciones virtuales; para zonas en donde predomina el clima soleado, por ejemplo Los Ángeles y Phoenix, el modelo de cielo despejado debe de ser considerado en los cálculos lumínicos.

Los modelos de cielo mencionados han sido ampliamente utilizados en las simulaciones de iluminación natural en todo el mundo (Kensek y Suk, 2011). Sin embargo, a pesar de las ventajas de la utilización de estas metodologías, su uso no se ha extendido. En este sentido, Reinhart y Wienold (2011) reportan que las razones son que su utilización implica un mayor tiempo y esfuerzo en comparación con el uso de la intuición, pero mucho menor que el cálculo manual matemático; otro factor relevante es el desconocimiento de los beneficios que de ellas derivan al facilitar el logro de un diseño adecuado, con niveles óptimos de iluminación natural muy precisa.

Se planteó el presente trabajo con el objetivo de proporcionar una visión de los aportes de la simulación de la iluminación natural en un espacio de oficina y para describir sus resultados a la luz de las normas para la eficiencia visua en consideración a variaciones estacionales, de orientación de fachada, de hora y del color a través de la utilización de los programas Radiance y Autodesk Ecotect.

Se compararon los niveles de iluminación natural (lux) en tres estaciones del año: primavera, verano e invierno; en dos horarios distintos: $12 \mathrm{HOO}$ y $18 \mathrm{HOO}$; dos orientaciones de fachada en la que se colocó una única ventana; y, por último, tres coeficientes de reflexión de las superficies -muros y techos-.

\section{Método}

\subsection{Características espaciales}

Para realizar el estudio se simuló un espacio de oficina, las dimensiones del espacio y del área de ventana (véase Tabla 1); se tomaron del Reglamento de Zonificación del Estado de Jalisco que marca como máximo un área de 20 metros cuadrados para oficinas y un área de ventana de 3 metros cuadrados.

El proyecto se situó en la ciudad de Guadalajara, bajo las siguientes coordenadas geográficas: latitud 20 $42^{\prime} 36^{\prime \prime} \mathrm{N}$ longitud 103 23' 24"; altitud de 1551 m.s.n.m. La Figura 3 muestra un corte longitudinal del espacio que se simuló.

\begin{tabular}{|l|l|}
\multicolumn{2}{|c|}{ Características del espacio } \\
\hline Dimensiones (m) & $4.0^{*} 5.0 * 2.30$ \\
\hline Volumen & $46 \mathrm{~m}^{3}$ \\
\hline Área & $20 \mathrm{~m}^{2}$ \\
\hline Muros & $\begin{array}{l}\text { De ladrillo espesor 11cm. } \\
\text { acabado enjarre pulido de } \\
1 \mathrm{~cm} . \text { Coeficiente de reflexión } \\
10 \%, 30 \%, 60 \% .\end{array}$ \\
\hline Piso & $\begin{array}{l}\text { Concreto 15 cm. de espesor } \\
\text { con acabado de baldosa } \\
\text { cerámica. Coeficiente de } \\
\text { reflexión 50\%. }\end{array}$ \\
\hline Techo & $\begin{array}{l}\text { Losa de concreto acabado de } \\
\text { enjarre pulido. Coeficiente de } \\
\text { reflexión 10\%, 60\%, 90\%. }\end{array}$ \\
\hline Orientación (ventana) & Sur - Norte \\
\hline Dimensiones (m) & $2.0 * 1.5$ \\
\hline Tipo de vidrio & Sencillo \\
\hline Espesor & $\begin{array}{l}6 \text { mm. } \\
\text { Sirea }\end{array}$ \\
\hline
\end{tabular}

Tabla 1: Características del espacio simulado 


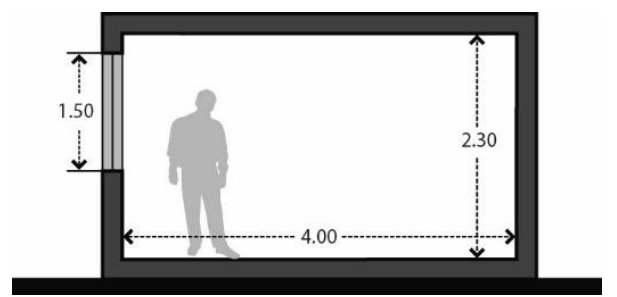

Figura 3: Sección longitudinal sin escala del espacio simulado.

\subsection{Simulación virtua}

El presente estudio se realizó a través de 36 simulaciones virtuales; el software seleccionado para realizar las simulaciones fue el Desktop Radiance V2. Este programa gratuito fue desarrollado para la realización de simulaciones lumínicas creado por el laboratorio de Berkeley. La utilización de este software se realizó a través de Autodesk Ecotec Analysis; en todos los casos, se simuló únicamente la iluminación natural. El modelo de cielo empleado fue el CIE despejado, debido a que este modelo de cielo considera la luz solar directa que ingresa a un espacio, elemento importante debido a la localización geográfica de la simulación.

Boyce (2003) indica que el criterio más utilizado de buena iluminación en una oficina es la iluminancia media, en un plano horizontal a la altura del escritorio; una superficie que convencionalmente se le llama plano de trabajo. Para realizar la simulación se trazó una retícula a la altura de plano de trabajo a $75 \mathrm{~cm}$. del nivel de piso terminado, de 10 por 10 obteniendo un total de 100 nodos; en cada uno se obtuvieron los valores correspondientes a las iluminancias.

Es importante mencionar que no se establecieron obstrucciones exteriores; es decir, elementos como edificios adicionales al simulado o vegetación así como tampoco se tomaron en cuenta las reflexiones del pavimento exterior. En la Figura 4 se muestra el diagrama de trayectoria solar con el espacio simulado en orientación Sur a las $12 \mathrm{H} 00$ en diciembre, así como la retícula situada a la altura del plano de trabajo.

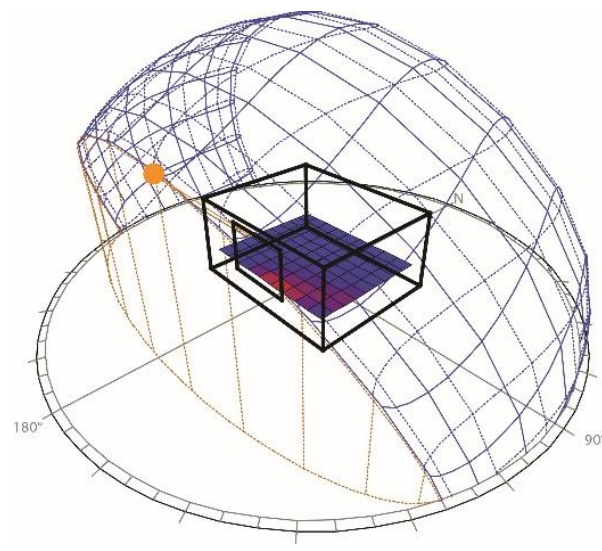

Figura 4: Diagrama de trayectoria solar y espacio simulado orientación sur a las $12 \mathrm{HOO}$ en invierno.

\subsection{Variables}

Las variables que se consideraron en las simulaciones para realizar las comparaciones fueron las descritas a continuación: se compararon tres temporadas distintas primavera (marzo), verano (junio) e invierno (diciembre), en dos horas diferentes a las $12 \mathrm{H} 00$ y a las $18 \mathrm{H} 00$; además, se contrastaron dos orientaciones de fachada en la que se situó una ventana al norte -18 simulaciones- y al sur -18 simulaciones- con el fin de comparar los niveles de iluminación según la orientación.

Otra variable fue el coeficiente de reflexión; para ello, se utilizaron tres combinaciones distintas (véase Tabla 2) aplicadas a las superficies interiores, techos y muros con e objetivo de comparar los niveles lumínicos obtenidos según el coeficiente de reflexión (color). En ningún caso se excedieron los valores máximos de reflexión establecidos en la Norma Oficial Mexicana: NOM-025-STPS-1999 condiciones de iluminación en los centros de trabajo (véase Tabla 3). Las dimensiones tanto del espacio como de la ventana permanecieron fijas.

\begin{tabular}{|c|c|c|c|c|}
\hline № & Elemento & $\begin{array}{c}\text { Reflexión } \\
\text { (\%) }\end{array}$ & Elemento & $\begin{array}{l}\text { Reflexión } \\
\text { (\%) }\end{array}$ \\
\hline 1 & Muros & 60 & Techo & 90 \\
\hline 2 & Muros & 30 & Techo & 60 \\
\hline 3 & Muros & 10 & Techo & 10 \\
\hline
\end{tabular}

Tabla 2: Combinaciones de coeficientes de reflexión en techos y muros.

\begin{tabular}{|l|c|}
\multicolumn{1}{|c|}{ Concepto } & $\begin{array}{c}\text { Niveles máximos permisibles de } \\
\text { reflexión }\end{array}$ \\
\hline Techo & $90 \%$ \\
\hline Paredes & $60 \%$ \\
\hline Piso & $50 \%$ \\
\hline Plano de trabajo & $50 \%$ \\
\hline
\end{tabular}

Tabla 3: Niveles máximos de reflexión permisibles según la Norma Oficial Mexicana NOM-025-STPS-1999, condiciones de iluminación en los centros de trabajo.

\section{Resultados}

Se obtuvieron dos tipos de resultados: iluminancias (lux) promedio del espacio simulado e iluminancias (lux) en seis puntos distintos del local. El primer punto fue a un metro de distancia a partir de la ventana y los siguientes cinco a 0.5 metros a partir del primer punto. En la Figura 5 se muestra una sección esquemática del espacio simulado, con la retícula a la altura del plano de trabajo en la que se representan los valores lumínicos obtenidos de la simulación; se puede observar que en las proximidades de la ventana se tienen los valores más altos de iluminancias y cómo estos valores disminuyen conforme nos aproximamos al fondo del local.

La Figura 6 muestra la planta del espacio simulado, en este caso corresponde a la orientación norte en verano y con el valor de reflexión de10\% tanto en muros como en el techo; además, se observa la retícula con los 100 nodos de los que se obtuvieron valores lumínicos. 

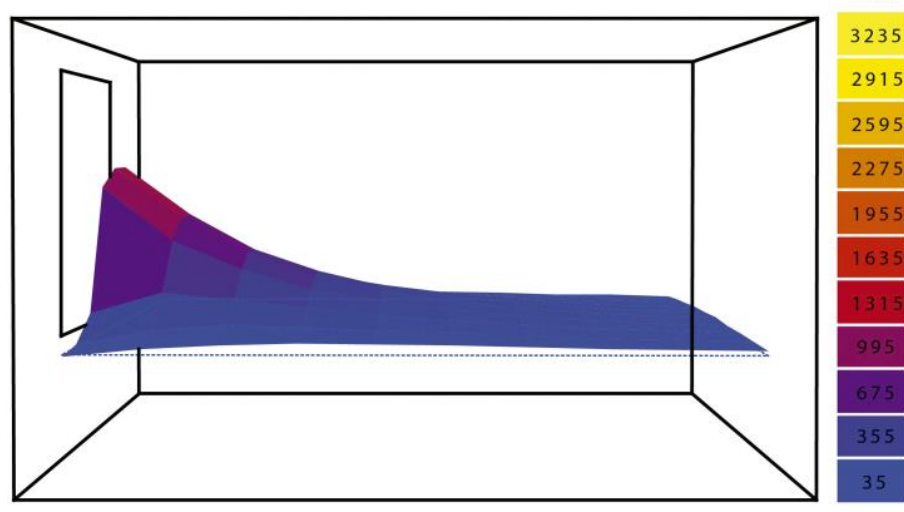

Figura 5: Sección esquemática longitudinal sin escala con retícula a la altura del plano de trabajo

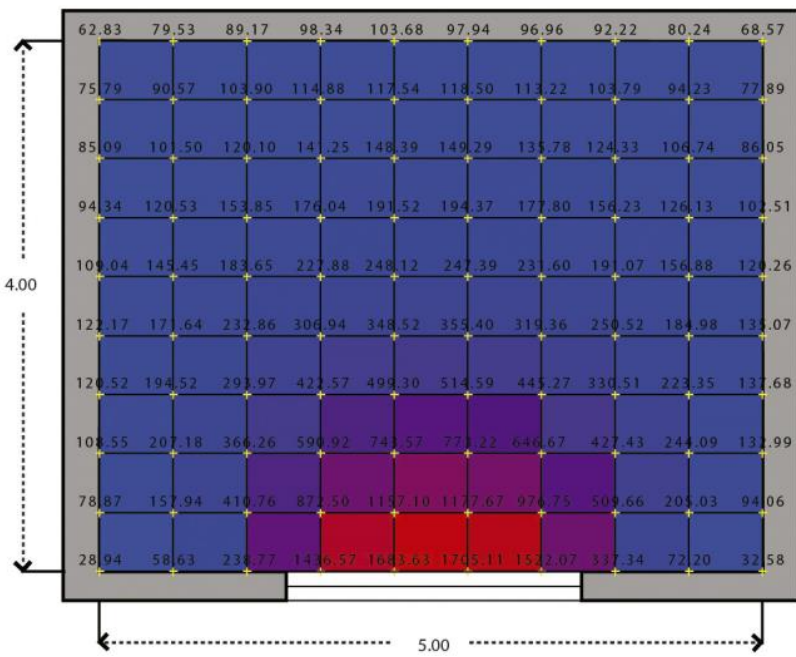

Figura 6: Planta con trazo de retícula del plano de trabajo a $75 \mathrm{~cm}$. del n.p.t con valores de iluminancias correspondientes a la simulación de junio, orientación norte, valor de reflexión $10 \%$ en muros y techo

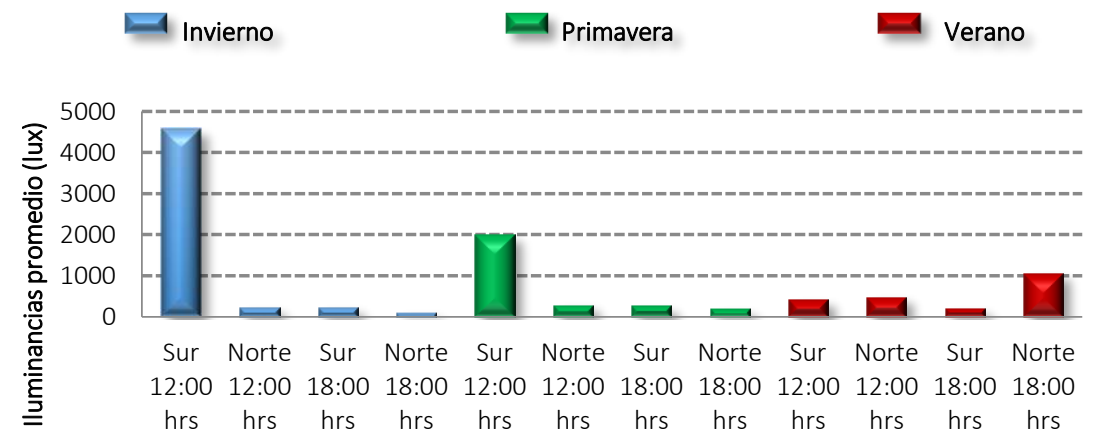

Figura 7: Comparación de iluminancias fachada norte y sur en primavera, verano e invierno con coeficiente de reflexión 10\% muros y $10 \%$ en techo

En la Figura 6 se observó que la simulación de la estación de invierno, con la orientación sur a las $12 \mathrm{HO0}$, es la que alcanza los niveles más altos de iluminación, superando hasta por 14 veces (véase Tabla 4) los 300 lux que marca la Norma Oficial mexicana de condiciones de iluminación en los centros de trabajo; para las $18 \mathrm{H} 00$ presentó los niveles más bajos 99 lux valor que no alcanza a estar dentro de la norma. También se observó que los niveles de iluminación de las épocas de invierno y primavera en las fachadas tanto norte como sur a las $18 \mathrm{HOO}$ y la fachada sur en verano, con el mismo horario, presentan datos similares estando por debajo de los 300 lux que exige la Norma. 


\begin{tabular}{|c|l|c|c|}
\hline Estación & Orientación & Hora & $\begin{array}{c}\text { Número } \\
\text { de veces }\end{array}$ \\
\hline \multirow{4}{*}{ Primavera } & Sur & $12 \mathrm{HOO}$ & 6 \\
\cline { 2 - 4 } & Norte & $12 \mathrm{HOO}$ & 0 \\
\cline { 2 - 4 } & Sur & $18 \mathrm{HOO}$ & 0 \\
\cline { 2 - 4 } & Norte & $18 \mathrm{HOO}$ & 0 \\
\hline \multirow{4}{*}{ Verano } & Sur & $12 \mathrm{HOO}$ & 0.4 \\
\cline { 2 - 4 } & Norte & $12 \mathrm{HOO}$ & 0.6 \\
\cline { 2 - 4 } & Sur & $18 \mathrm{HOO}$ & 0.3 \\
\cline { 2 - 4 } & Norte & $18 \mathrm{HOO}$ & 2.6 \\
\hline \multirow{3}{*}{ Invierno } & Sur & $12 \mathrm{HOO}$ & 14.3 \\
\cline { 2 - 4 } & Norte & $12 \mathrm{HOO}$ & -0.2 \\
\cline { 2 - 4 } & Sur & $18 \mathrm{HOO}$ & -0.2 \\
\cline { 2 - 4 } & Norte & $18 \mathrm{HOO}$ & -0.7 \\
\hline
\end{tabular}

Tabla 4: Veces que se supera el nivel de iluminación con referencia a la NOM-025-STPS-1999

En la Tabla se muestra el número de veces que es superado el nivel de iluminación establecido por la NOM-025-STPS1999, en la simulación realizada con coeficiente de reflexión de $10 \%$ en muros y $10 \%$ en techo.

$\leq 0$ indica valores menores a 300 luxes; $0=300$ luxes; $\geq 1$ indica valores mayores a 300 .

En la Figura 8 se muestran los resultados correspondientes a la simulación en la que se aplicaron valores de $30 \%$ de reflexión en muros y $60 \%$ en techo. Esta simulación demostró que la orientación sur, en invierno a las $12 \mathrm{HOO}$, es la de mayor iluminación en el espacio interior, superando por 23 veces los 300 lux establecidos por la Norma. En el extremo contrario, se encontró con los niveles lumínicos más bajos (123 lux) en la fachada norte de la temporada invernal, a las 18H00; en este caso, las fachadas norte y sur en la época de primavera a las $18 \mathrm{HOO}$ alcanzaron 256 y 247 luxes respectivamente. En la época de verano ambas fachadas y horarios presentan niveles homogéneos; es decir, es la época en que se presenta menor variabilidad de lux.

En la Tabla 5 se observa que en la época de verano, en todos los casos simulados, se alcanza a superar los niveles mínimos que exige la Norma.

\begin{tabular}{|c|l|c|c|}
\hline Estación & Orientación & Hora & $\begin{array}{c}\text { Número } \\
\text { de veces }\end{array}$ \\
\hline \multirow{4}{*}{ Primavera } & Sur & $12 \mathrm{HOO}$ & 7.2 \\
\cline { 2 - 4 } & Norte & $12 \mathrm{HOO}$ & 0.7 \\
\cline { 2 - 4 } & Sur & $18 \mathrm{HOO}$ & -0.2 \\
\cline { 2 - 4 } & Norte & $18 \mathrm{HOO}$ & -0.1 \\
\hline \multirow{4}{*}{ Verano } & Sur & $12 \mathrm{HOO}$ & 1.3 \\
\cline { 2 - 4 } & Norte & $12 \mathrm{HOO}$ & 1.5 \\
\cline { 2 - 4 } & Sur & $18 \mathrm{HOO}$ & 0 \\
\cline { 2 - 4 } & Norte & $18 \mathrm{HOO}$ & 3.2 \\
\hline \multirow{4}{*}{ Invierno } & Sur & $12 \mathrm{HOO}$ & 23 \\
\cline { 2 - 4 } & Norte & $12 \mathrm{HOO}$ & 0.4 \\
\cline { 2 - 4 } & Sur & $18 \mathrm{HOO}$ & -0.6 \\
\cline { 2 - 4 } & Norte & $18 \mathrm{HOO}$ & -0.6 \\
\hline
\end{tabular}

Tabla 5: Veces que se supera el nivel de iluminación respecto a la NOM-025-STPS-1999

En la Tabla se muestra el número de veces que es superado el nivel de iluminación establecido por la NOM-025-STPS1999, en la simulación realizada con coeficiente de reflexión de $30 \%$ en muros y $60 \%$ en techo.

$\leq 0$ indica valores menores a 300 luxes; $0=300$ luxes; $\geq 1$ indica valores mayores a 300 .

Por último, en lo correspondiente a iluminancias promedio (véase Figura 9), en este caso simulado con el coeficiente de reflexión de $60 \%$ en muros y $90 \%$ en el techo, al igual que en las situaciones anteriores, los resultados mostraron que los niveles máximos de iluminación corresponden a la estación de invierno con orientación sur a las $12 \mathrm{HOO}$ superando por 27 veces las exigencias lumínicas de la Norma Mexicana. En todas las situaciones, con excepción de invierno con fachada norte a las $18 \mathrm{HOO}$, en las simulaciones realizadas con los coeficientes de reflexión máximos permisibles, se superó el nivel de 300 luxes (NOM-025-STPS-1999).

La diferencia de los niveles lumínicos de la época de invierno en la fachada sur a las $12 \mathrm{HOO}$, con relación a las $18 \mathrm{HOO}$ representa un $92.4 \%$ mientras que en la fachada norte esta diferencia representa el $73.8 \%$. Por otro lado, el caso de la época de verano la diferencia de luxes entre la fachada sur a las $12 \mathrm{HOO}$ y a las $18 \mathrm{HOO}$ representa un $53.4 \%$ mientras que en la misma época en la fachada norte, los valores de las $18 \mathrm{HOO}$ respecto a los de las $12 \mathrm{HOO}$ representan un $35.2 \%$.

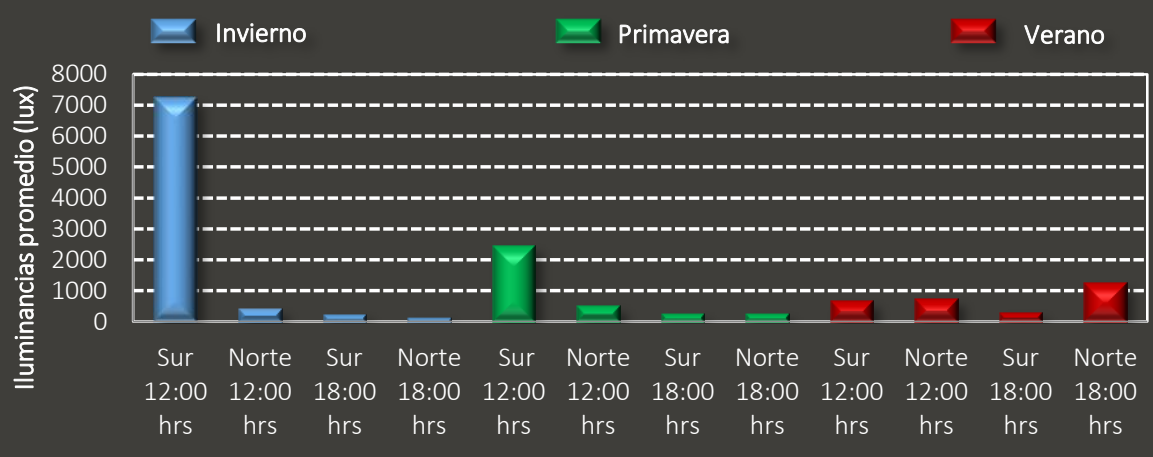




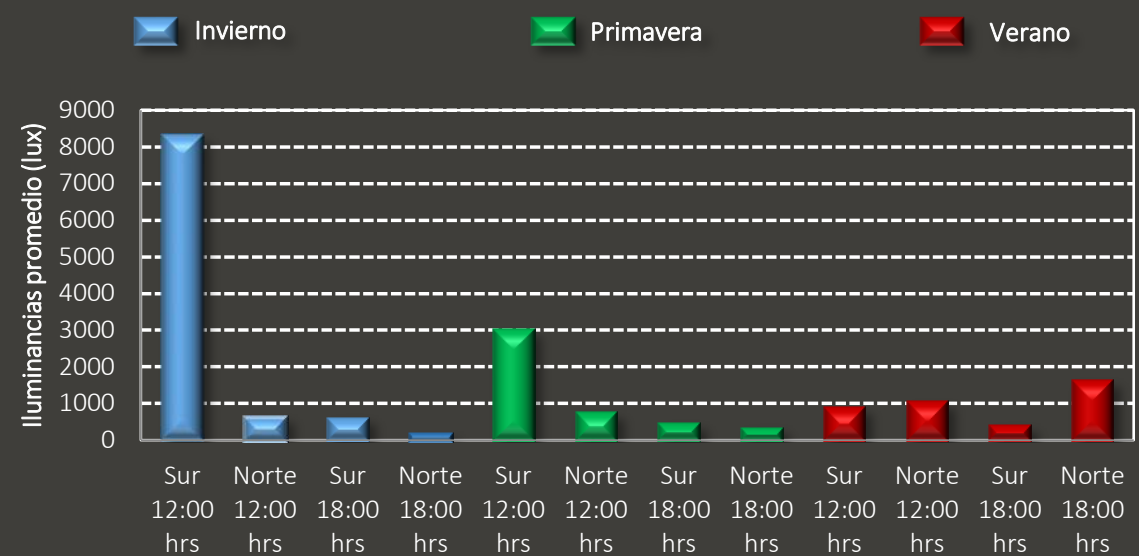

Figura 9: Comparación de iluminancias fachada norte y sur en primavera, verano e invierno con coeficiente de reflexión 60\% muros y $90 \%$ en techo

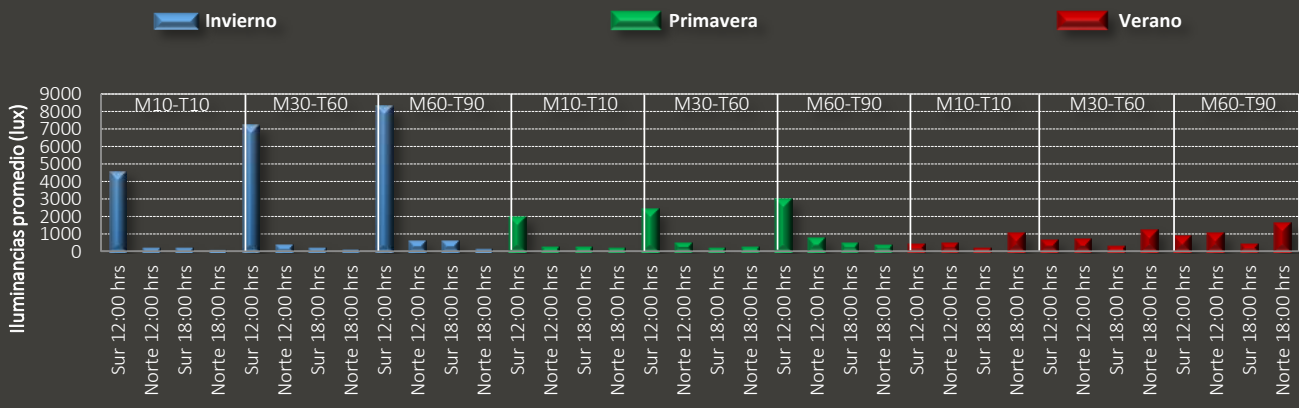

Figura 10: Comparación de iluminancias promedio en fachada norte y sur en primavera, verano e invierno; $12 \mathrm{HOO}$ y $18 \mathrm{HOO}$ y tres combinaciones de coeficiente de reflexión.

\begin{tabular}{|c|l|c|c|}
\hline Estación & Orientación & Hora & $\begin{array}{c}\text { Número } \\
\text { de veces }\end{array}$ \\
\hline \multirow{4}{*}{ Primavera } & Sur & $12 \mathrm{HOO}$ & 9.1 \\
\cline { 2 - 4 } & Norte & $12 \mathrm{HOO}$ & 1.6 \\
\cline { 2 - 4 } & Sur & $18 \mathrm{HOO}$ & 0.6 \\
\cline { 2 - 4 } & Norte & $18 \mathrm{HOO}$ & 0.2 \\
\hline \multirow{4}{*}{ Verano } & Sur & $12 \mathrm{HOO}$ & 2.0 \\
\cline { 2 - 4 } & Norte & $12 \mathrm{HOO}$ & 2.6 \\
\cline { 2 - 4 } & Sur & $18 \mathrm{HOO}$ & 0.4 \\
\cline { 2 - 4 } & Norte & $18 \mathrm{HOO}$ & 4.5 \\
\hline \multirow{4}{*}{ Invierno } & Sur & $12 \mathrm{HOO}$ & 26.8 \\
\cline { 2 - 4 } & Norte & $12 \mathrm{HOO}$ & 1.1 \\
\cline { 2 - 4 } & Sur & $18 \mathrm{HOO}$ & 1.1 \\
\cline { 2 - 4 } & Norte & $18 \mathrm{HOO}$ & -0.5 \\
\hline
\end{tabular}

Tabla 6: Veces que se supera el nivel de iluminación respecto a la NOM-025-STPS-1999

En la Tabla se muestra el número de veces que es superado el nivel de iluminación establecido por la NOM-025-STPS1999, en la simulación realizada con coeficiente de reflexión de $60 \%$ en muros y $90 \%$ en techo.

$\leq 0$ indica valores menores a 300 luxes; $0=300$ luxes; $\geq 1$ indica valores mayores a 300 .
Con el objetivo de facilitar la comparación de los resultados obtenidos, en la Figura 10 se muestran agrupados por época (primavera, verano e invierno) los resultados de las tres combinaciones de coeficientes de reflexión que se aplicaron a las superficies de muros y techos, los dos horarios simulados $(12 \mathrm{HOO}$ y $18 \mathrm{HOO})$ y las dos orientaciones (norte y sur).

Los valores más altos se presentaron en la época de invierno en la orientación sur a las 12H00; es también en esta época en donde se encontraron variaciones más drásticas de los niveles lumínicos en relación a las fachadas norte y sur. En segundo lugar, los valores más altos se presentaron en la época de primavera, en la orientación sur a las $12 \mathrm{HOO}$; y, por último, en verano en orientación norte a las $12 \mathrm{HOO}$ se registraron las variaciones mínimas entre orientaciones y horarios.

En la Figura 10 se observó que aumentan los valores lumínicos cuando se aumenta el coeficiente de reflexión; esta diferencia representó hasta un $45 \%$ más luxes en un escenario con las misma condiciones variando tan sólo e coeficiente de reflexión, en el caso de la fachada sur. En la orientación norte, con las mismas condiciones, varia e coeficiente de reflexión; la diferencia representó $63 \%$. 
En relación a los resultados que se obtuvieron en seis puntos distintos del espacio interior, se puede observar en la Figura 11 que el espacio simulado con la ventana en la fachada sur, a las $12 \mathrm{HOO}$, superó por $55.9 \%$ el nivel de luxes (1684) respecto a la simulación con la ventana dispuesta en la fachada norte (743 lux); lo anterior a un metro de distancia de la ventana mientras el punto más alejado de la ventana $(3.50 \mathrm{~m})$ registró $43.5 \%$ más iluminación.

Al comparar los niveles lumínicos sólo por la orientación norte y sur- pero conservando la hora $12 \mathrm{H} 00$, la diferencia entre las $12 \mathrm{HOO}$ y las $18 \mathrm{HOO}$ representa un $45 \%$ a un metro de distancia de la ventana; el mismo escenario pero a 3.5 metros de distancia, la diferencia representa un $41 \%$. En el caso de la orientación sur, la diferencia de niveles lumínicos entre las $12 \mathrm{H0O}$ (1684 lux) y las $18 \mathrm{HOO}$ (495 lux) representa el $71 \%$ a un metro de distancia de la ventana mientras que a 3.5 metros de distancia representa un $65 \%$. Todos los casos descritos anteriormente corresponden a la temporada de primavera, con un coeficiente de reflexión de $10 \%$ tanto en muros como en el techo.

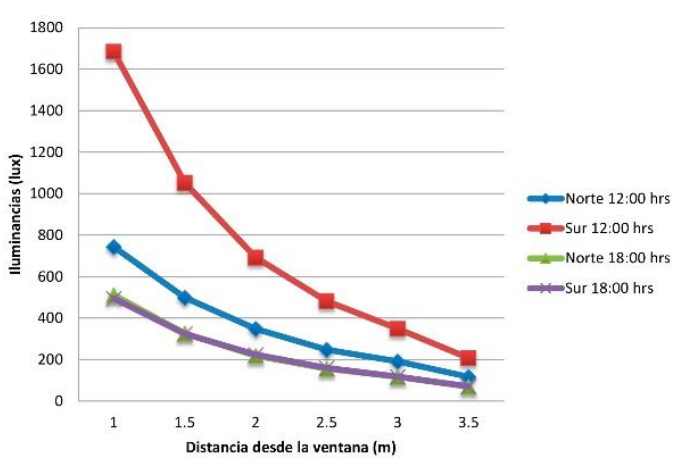

Figura 11: Comparación de iluminancias fachada norte y sur en primavera, con coeficiente de reflexión $10 \%$ muros y $10 \%$ en techo.

En el caso del escenario correspondiente a la época de verano con coeficientes de reflexión del $10 \%$ en muros y techo, la Figura 12 muestra que la fachada con orientación norte superó ligeramente (8\%) los niveles de iluminación, respecto a la fachada con orientación sur a un metro de distancia a las 12H00; a 3.5 metros de distancia a partir de la ventana de la fachada norte supera únicamente por $4 \%$ a la fachada sur a las $12 \mathrm{HOO}$.

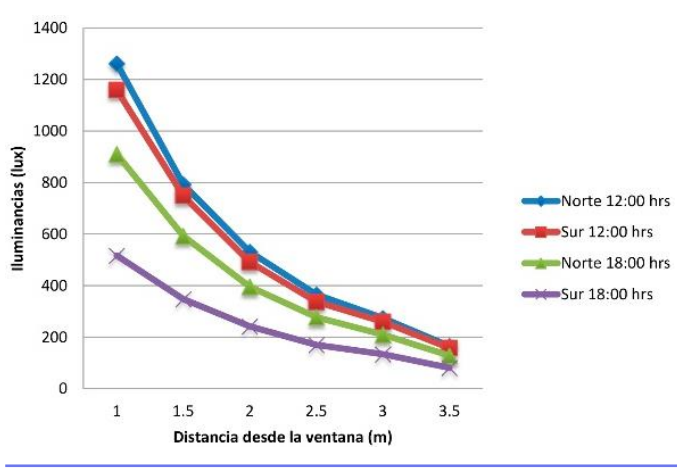

Figura 12: Comparación de iluminancias fachada norte y sur en verano, con coeficiente de reflexión $10 \%$ muros y $10 \%$ en techo.
En cuanto a la comparativa por horarios, considerando la misma orientación la fachada norte a las $12 \mathrm{HOO}$ a un metro de distancias representa un $38.5 \%$ más de iluminación respecto a las $18 \mathrm{HOO}$. En la fachada sur la diferencia de los niveles lumínicos a un metro de distancia desde la ventana respecto a los horarios $12 \mathrm{HOO}$ (1116 lux) y $18 \mathrm{H00}$ (517 lux) representa un $55.4 \%$.

Finalmente, la época de invierno (véase Figura 13) con valores de 10\% de reflexión en la fachada sur reportó 37198 luxes $(12 \mathrm{HOO})$ y superó por mucho los valores de la fachada norte, la cual reportó 643 luxes $(12 \mathrm{HOO})$ en el punto más cercano a la ventana (1 metro); sin embargo, en los datos correspondientes al punto más alejado de la ventana, los niveles lumínicos con la ventana en la fachada sur descendieron de forma considerable, registraron 346 lux; aun así continúa superando los niveles registrados en la orientación norte (107 lux) representando un 69\% dicha diferencia. En esta época, la diferencia que se encontró al comparar los horarios $12 \mathrm{HOO}$ y $18 \mathrm{HOO}$ en orientación norte corresponde a un $59.7 \%$ mientras que en la orientación sur representa un $100 \%$. Los casos anteriores corresponden a los valores obtenidos a un metro de distancia desde la ventana.

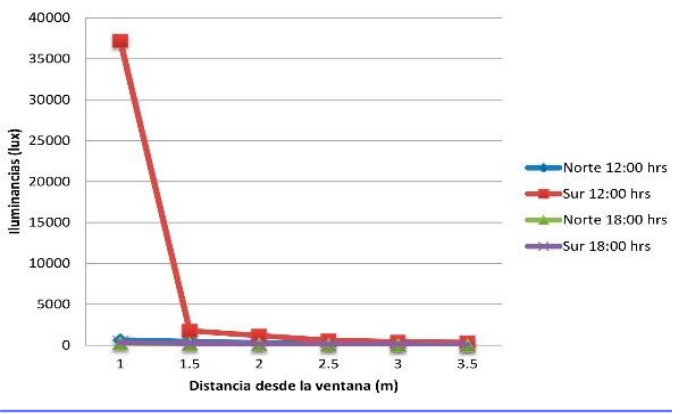

Figura 13: Comparación de iluminancias, fachada norte y sur, en invierno con coeficiente de reflexión 10\% muros y 10\% en techo.

Los resultados obtenidos del escenario en el que se aplicó $30 \%$ de reflexión en muros y $60 \%$ en el techo determinaron que la diferencia obtenida entre los niveles máximos de iluminación (fachada sur 12HOO) a un metro de distancia desde la ventana representaron un $54.6 \%$ más que los niveles registrados en la orientación norte a las $12 \mathrm{HOO}$ (véase Figura 14).

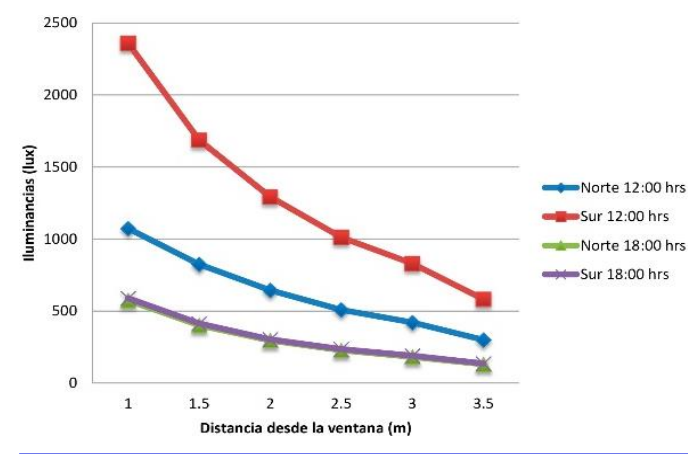

Figura 14: Comparación de iluminancias de la fachada norte y sur en primavera con coeficiente de reflexión 30\% muros y $60 \%$ en techo. 
La diferencia de niveles lumínicos en la orientación norte a las $12 \mathrm{HOO}$ representa un $46.6 \%$ más respecto a los valores obtenidos a las $18 \mathrm{H} 00$ en relación a la fachada sur; la diferencia de luxes de las $12 \mathrm{HOO}$ en relación a las $18 \mathrm{HOO}$ representa un $75 \%$. Estos datos corresponden a un metro de distancia desde la ventana.

Con respecto a los resultados correspondientes a la época de verano, la Figura 15 muestra que la orientación norte supera en un $8 \%$ los niveles lumínicos de la orientación sur esto a un metro de distancia de la ventana a las $12 \mathrm{HOO}$ y a 3.5 metros de distancia de la ventana este valor desciende a $4 \%$. En cuanto a la orientación norte la diferencia de luxes a las $12 \mathrm{HOO}$ representó un $34.8 \%$ más que los valores registrados a las $18 \mathrm{HOO}$ a un metro de distancia desde la ventana; este porcentaje se mantuvo constante al fondo del local a 3.5 metros de distancia desde la ventana. A las $12 \mathrm{HOO}$ se registraron 390 luxes mientras que a las $18 \mathrm{H} 00$ se registraron 250 luxes que representó un 36\% más iluminación que a las $12 \mathrm{HOO}$. Por otro lado, en la orientación sur se registraron 1538 luxes a las $12 \mathrm{HOO}$ y 640 luxes a las 18H00; esto implicó $58.3 \%$ más luxes, estos datos corresponden a un metro de distancia desde la ventana.

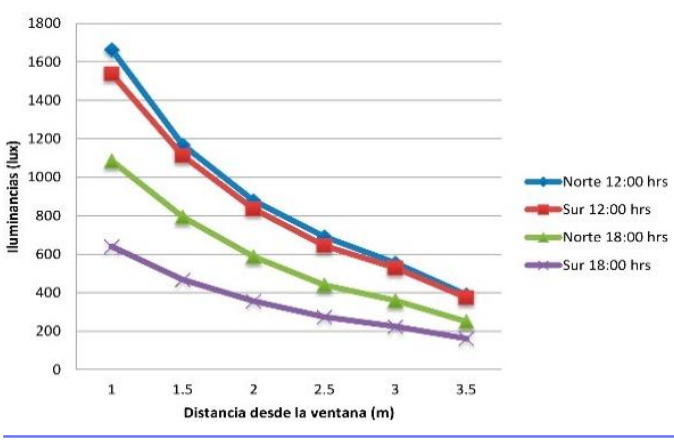

Figura 15: Comparación de iluminancias en la fachada norte y sur en verano, con coeficiente de reflexión 30\% muros y $60 \%$ en techo.

La Figura 16 muestra los valores obtenidos de la simulación de invierno para los coeficientes de reflexión de $30 \%$ en muros y $60 \%$ en techo, en la cual se registraron 38435 luxes a un metro de distancia, desde la ventana en la orientación sur mientras que en la orientación norte se registraron 900 luxes; en uno de los punto más alejados de la ventana (3.5 metros) la orientación sur registró 1252 lux y la fachada norte 264. Estos datos corresponden a las $12 \mathrm{HOO}$

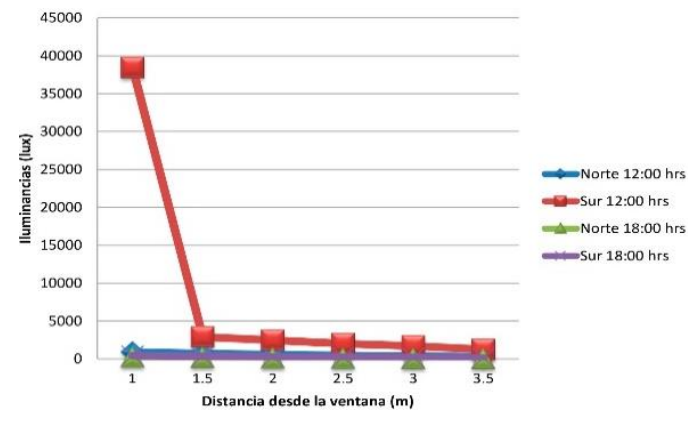

Figura 16: Comparación de iluminancias en las fachadas norte y sur en invierno, con coeficiente de reflexión 30\% muros y $60 \%$ en techo.
Los siguientes resultados pertenecen a la combinación tres de coeficientes de reflexión que corresponden a $90 \%$ para e techo y $60 \%$ para muros; esto como se mencionó con anterioridad son los valores máximos permisibles por la NOM-025-STPS-1999.

En el caso de la época de primavera la Figura 17 muestra que la fachada sur, en el punto más cercano a la ventana (a un metro de distancia) aportó un $54.1 \%$ más iluminación natural con respecto a la fachada sur; mientras en el punto más alejado (3.5 metros), la orientación sur contribuyó un $50 \%$ más de los niveles lumínicos respecto a la orientación norte a las $12 \mathrm{HOO}$. En la orientación norte a un metro de distancia, desde la ventana, a las 12:00 se registraron 1635 luxes mientras que a las $18 \mathrm{HO0}, 677$ luxes; esto representó $50.4 \%$ más iluminación. Con los mismo parámetros, pero al fondo del local (3.5 metros desde la ventana) esta diferencia representa un $60 \%$ más iluminación a las $12 \mathrm{HOO}$ con respecto a las $18 \mathrm{HOO}$. Por otro lado, en la fachada sur la diferencia a un metro de distancia representó $76.7 \%$ más luxes a las $12 \mathrm{HOO}$ en relación a las $18 \mathrm{HOO}$.

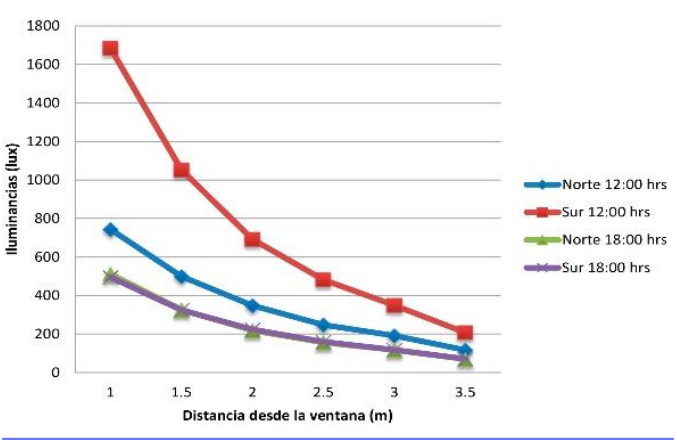

Figura 17: Comparación de iluminancias de las fachada norte y sur en primavera, con coeficiente de reflexión $60 \%$ muros y $90 \%$ en techo.

Con respecto a la época de verano, en la Figura $\mathrm{N}^{\circ} 18$ podemos observar que la diferencia de niveles lumínicos entre las orientaciones norte y sur es mínima.

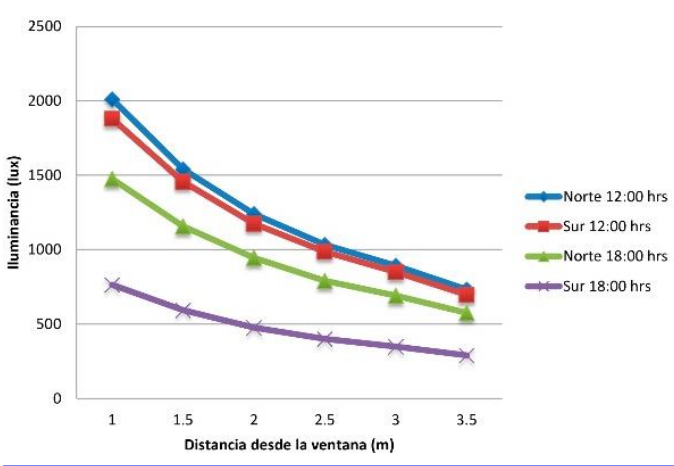

Figura 18: Comparación de iluminancias n las fachadas norte y sur en verano, con coeficiente de reflexión $60 \%$ muros y $90 \%$ en techo.

La orientación norte registró 2000 luxes y la sur 1800 luxes a un metro de distancia desde la ventana; esto corresponde a un $6 \%$ de diferencia entre ambas orientaciones a las $12 \mathrm{H00}$; y, en el punto más alejado de la ventana (3.5 metros) este 
porcentaje disminuye a 5\%. La iluminación aumentó 36\% a las $12 \mathrm{HOO}$ (2011 lux) con respecto a los valores de las $18 \mathrm{HOO}$ (1476) en la orientación norte a un metro de distancia; en el mismo escenario, pero a 3.5 metros de distancia, esta diferencia representa $21 \%$. Los valores correspondientes a la orientación sur a las $12 \mathrm{H} 00$ representan $59.4 \%$ más que los valores obtenidos a las $18 \mathrm{HOO}$.

Por último, los valores obtenidos de la época de invierno se representaron en la Figura 19 en donde se observó que la orientación sur superó por mucho los valores lumínicos de la orientación norte. En el caso de la simulación con la ventana dispuesta en la orientación sur se registraron 39498 luxes y en la orientación norte se registraron 1132 luxes a un metro de distancia desde la ventana a las $12 \mathrm{HOO}$; sin embargo, en el punto más alejado de la ventana, es decir a 3.5 metros, los niveles de la orientación sur descienden drásticamente en comparación con el punto más cercano, registrando 2401 luxes mientras la orientación norte registró 483 luxes a las $12 \mathrm{HOO}$

La orientación norte a las $12 \mathrm{HOO}$ registró $71.3 \%$ más iluminación que los valores obtenidos a las $18 \mathrm{HOO}$, a un metro de distancia; en la orientación sur la diferencia de niveles lumínicos respecto a la hora es prácticamente de $100 \%$, registrando 39498 lux a las $12 \mathrm{H} 00$ y 645 luxes a las $18 \mathrm{HOO}$

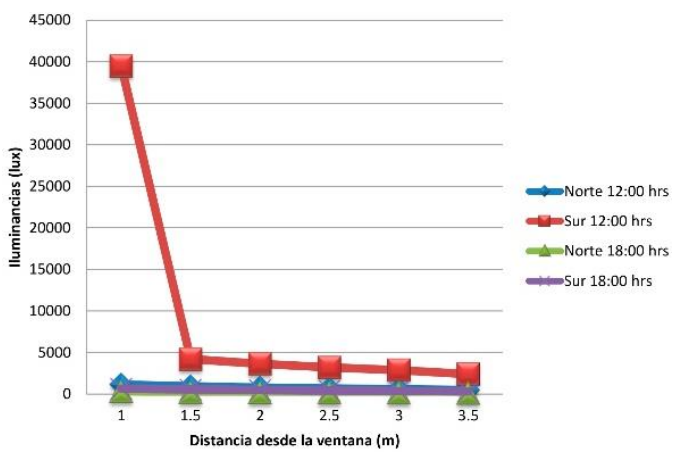

Figura 19: Comparación de iluminancias de las fachada norte y sur, en invierno, con coeficiente de reflexión $60 \%$ muros y $90 \%$ en techo.

\section{Conclusiones}

Es bien conocido que las propiedades de reflexión del color influyen en los niveles de iluminación: mientras mayor sea e coeficiente de reflexión, el nivel de iluminación aumentará Sin embargo, los resultados obtenidos en este trabajo demostraron cómo se puede variar en gran magnitud el nive de iluminación, tan sólo con la adecuada selección del color en muros y techo. Por ejemplo, en el caso de la orientación sur en invierno a las $12 \mathrm{H00}$, con un coeficiente de reflexión de $10 \%$ se obtuvieron 4583 lux promedio, mientras que en el mismo caso tan sólo cambiando la variable del coeficiente de reflexión por $60 \%$ en muros y $90 \%$ en techo se obtuvieron 8347 lux en promedio; esto representa un $45 \%$ más de luxes en el espacio interior.

Todos los casos simulados con los coeficientes de reflexión más altos alcanzaron e inclusive superaron los niveles de iluminación exigidos por la NOM-025-STPS-1999 (300 luxes) con excepción de la orientación norte a las $18 \mathrm{H} 00$. No obstante, es importante resaltar que desde el punto de vista ergonómico, el rango de iluminación para trabajo en oficina va desde 200 a 500 luxes de acuerdo a la tarea porque para trabajo en computadora 200 luxes puede ser suficiente, pero para leer-actividad que también se lleva a cabo en una oficina- el requerimiento de iluminación se eleva (Prado León et. al, 2009).

Es importante mencionar que aunque para la norma oficia estos coeficientes de reflexión son los más altos permitidos -no especifica los mínimos aceptables- para lograr una eficiencia visual; los ergónomos recomiendan que el techo tenga un coeficiente de reflexión de 80-90, los muros de 40 60 y el piso de 20-40 luxes. También es relevante e coeficiente de reflexión de la maquinaria y equipo (30-50 luxes) y de la superficie de trabajo (20-45 luxes) para que no cause deslumbramiento o incomodidad visual (Prado León, Ávila Chaurand, Aceves González, González Muñoz, 2009).

Con respecto a las simulaciones con los coeficientes de reflexión de $30 \%$ en muros y $60 \%$ en techos, cinco casos no alcanzaron los niveles de iluminación que exige la norma. En las simulaciones con el coeficiente de reflexión más bajo (10\% en muros y techos) siete casos no lograron alcanzar los niveles establecidos por la norma; por tanto, a pesar de que el color no es la única variable a considerar para crear un ambiente lumínico óptimo, sí es un elemento de suma importancia que deberá considerarse en el diseño.

Como se pudo observar en los resultados, los niveles de iluminación varían considerablemente según la orientación en la cual se encuentre dispuesta la ventana, así como la época del año y la hora. En el presente trabajo se obtuvieron los niveles más altos, en todos los casos, en la época de invierno al sur a las $12 \mathrm{HOO}$ y los más bajos al norte en época de invierno (diciembre) a las $18 \mathrm{H00}$; esto se debe a la altitud y el azimut del sol en cada época y hora, en relación a la localización geográfica.

Por tanto, el arquitecto y diseñador de interiores deberá estar familiarizado con la geometría solar de la localidad en donde se realice el proyecto; esto le permitirá diseñar de acuerdo a dichas condicionantes y crear ambientes más ergonómicos.

De las 36 simulaciones realizadas, 12 no alcanzaron los 300 luxes; en su mayoría, aquellos que se realizaron con el coeficiente de reflexión de $10 \%$ en muros y techo y todos a las $18 \mathrm{H} 00$. Estos datos indican que se podría iluminar de manera natural la mayor parte de una jornada laboral.

Por otro lado, es importante aclarar que no siempre tener niveles altos de iluminación será sinónimo de un espacio más confortable porque puede conducir a deslumbramiento y generación de calor extremo, pues la luz es una energía que se trasformará en calor y, según sea el caso, será o no beneficioso en el confort térmico y lumínico del usuario y por ende, podría afectar en forma negativa la eficiencia y confort visual. Por esto, en el campo de la ergonomía, también se señalan los niveles máximos de luxes y no sólo el mínimo requerido como en la norma oficial.

En este sentido, de acuerdo a los resultados obtenidos en la simulación, sobre todo en las orientaciones sur, a las $12 \mathrm{H00}$, aplicando los niveles máximos de reflexión, los niveles 
obtenidos (por ejemplo con 30\% de reflexión en muros y $60 \%$ en techos, se obtuvo casi 7000 luxes) podrían ser excesivos, provocando los efectos antes descritos. Por tanto, en ciertas situaciones y en consideración a la localización geográfica del proyecto así como a la orientación de aberturas se necesitará proteger del exceso de luz; esto se podría hacer por medio de sistemas de protección solar tratamientos en fachadas e inclusive estudiando la dimensión de las aberturas.

Si bien en este trabajo se utilizó el modelo de cielo despejado, debido al clima predominantemente soleado de Guadalajara, es importante considerar que en situaciones como la temporada de lluvias cuando se presentan días nublados, no se alcanzarán los niveles de iluminación mostrados en este documento; por tanto, el modelo de cielo despejado arroja datos del "mejor de los escenarios" que podría considerarse, existe la mayor parte del año.

Es importante señalar que la posición de la abertura por donde ingresará la luz natural y su proporción sería otra variable interesante a analizar. Además de los beneficios psicológicos y biológicos que proporciona la iluminación natural a los usuarios así como el tener vista hacia fuera, otro tema interesante a explorar es el energético. En este sentido, diseñar espacios con luz natural a más de los efectos positivos en la eficiencia visual y confort del usuario se disminuirían los consumos energéticos, aportando a la sustentabilidad.

Otro punto a considerar es que, en este estudio, se comparó el nivel lumínico de acuerdo a variaciones en la orientación el coeficiente de reflexión, la estación del año y la hora; sin embargo, no se consideraron otros aspectos importantes de ambiente lumínico como el deslumbramiento, luminancias y contrastes que deberán tomarse en cuenta a la hora de diseñar un espacio arquitectónico. Se sugiere realizar estudios que incluyan nuevas posibilidades de simulaciones de iluminación que consideren dichos tópicos.

Con referencia a las relaciones de contraste recomendadas en el ambiente de trabajo, se ha reportado que demasiado contraste entre las áreas visuales centrales y periféricas es incómodo e interfiere con la visión y el trabajo. La brillantez del área de trabajo no debe superar más de tres veces la de los alrededores cercanos y no más de diez la de los alrededores lejanos (Kroemer y Grandjean, 2001).

Finalmente es relevante hacer notar que los resultados de la simulación contemplaron la ventana desnuda, sin considerar ninguna protección como podría ser una cortina. Aunque es importante que el usuario tenga la posibilidad de tener visión hacia fuera, también en ocasiones puede requerir aminorar el nivel de iluminación o la entrada del sol. En este sentido, la recomendación general es que se seleccione la combinación de factores considerados en la simulación que arroje un nivel superior al requerido porque el resguardo evidentemente bajará los niveles de iluminación, según sea el elemento protector.

A través de los resultados alcanzados, el trabajo se enfocó en proporcionar elementos para aumentar el conocimiento y uso de las simulaciones como una potente herramienta para el análisis del proyecto en etapas tempranas del diseño. Este conocimiento puede contribuir a determinar las estrategias arquitectónicas necesarias para lograr niveles óptimos de iluminación, al considerar las variables mencionadas en este estudio para obtener espacios de trabajo en donde el usuario se beneficie de los atributos de la iluminación natural tales como: seguridad, confort y productividad, entre otros. 


\section{Bibliografía}

- Aguirre, F. E., \& Cuvilier, M. "Efectos del color en los espacios interiores". G. Boyce, P., (2003). Human Factors in Lighting. Londres, Inglaterra: Taylor \& Francis Group, 2014.

- Boyce, P. Illumination: basic definition. International. Encyclopedia of Ergonomics and Human Factors. Karwowski W. edit. (2011): 10161021. New York: Taylor \& Francis, 2011.

- Boyce, P., Hunter, C. \& Howlett, O. The benefits of daylight through windows. Troy, New York: Rensselaer Polytechnic Institute, 2003.

- Fuentes Romero, J. J. "El edificio de la biblioteca de Viipuri: Alvar Aalto, el humanismo innovador de un hacedor de bibliotecas". Anales de documentación 3 (2000): 67-79.

- Kaplan, R., \& Dana, S. T. "The nature of the view from home: Psychological benefits". Enviorimental and Behavior 33.4 (2001): 507-245

- $\quad$ Kensek, K. \& Suk, J. Y. "Daylight Factor (overcast sky) versus Daylight Availability (clear sky) in Computerbased Daylighting Simulations". Journal of Creative Sustainable Architecture \& Built Environment, 1 (2011): 3-14

- Kliczkowski H. (2002). Alvar Aalto. Madrid, España: Loft Publications, 2002.

- $\quad$ Kroemer, K. H. E. y Grandjean, E. "Fitting the task to the human". A textbook of occupational ergonomics. Londres: Taylor \& Francis, 2001.

- $\quad$ Li, D. H., Cheung, G. H., Cheung, K. L., \& Lam, T. N. "Determination of vertical daylight illuminance under non-overcast sky conditions". Building and environment 45.2 (2010): 498-508.

- $\quad$ Li, D. H., \& Tsang, E. K. "An analysis of measured and simulated daylight illuminance and lighting savings in a daylit corridor". Building and Environment, 40.7 (2005): 973-982

- $\quad$ Li, D. H., Wong, S. L., Tsang, C. L., \& Cheung, G. H. (2006). "A study of the daylighting performance and energy use in heavily obstructed residential buildings via computer simulation techniques". Energy and Buildings 38 (2006): 13431348.

- Nussbaumer, L. Human Factors in the Built Enviroment. Nueva York, Estados Unidos: Fairchild Books, 2014

- Ortiz, G. Uso, aplicaciones y creencias acerca del color. México, D.F.: Trillas, 2004.

- Ortiz \& B. Solórzano (ed.). Tópicos del Color en México y el mundo. México, D.F: Universidad Autónoma Metropolitana Unidad Azcapotzalco, 2014

- Prado León Lilia R., Ávila Chaurand R. Aceves González C. y González Muñoz E. Trabajo Con Computadoras. Recomendaciones Para la Salud, Comodidad y Productividad. Guadalajara, México: Universidad de Guadalajara, 2009.

- Reinhart, C. F., \& Wienold, J. "The daylighting dashboard. A simulation-based design analysis for daylit spaces". Building and environment, 46.2 (2011): 386-396.

- Resene. "The paint the professionals use", 2015. 1 de julio de 2015 http://www.resene.co.nz/swatches/index.php) Secretaria del Trabajo y Prevención Social, 2015. 3 junio de 2015 http://webcache.googleusercontent.com/search?q =cache:gfRyDTgJsDgJ:www.stps.gob. $\mathrm{mx} / \mathrm{bp} /$ seccio nes/dgsst/normatividad/normas/Nom025.pdf $+\& \mathrm{~cd}=1 \& \mathrm{hl}=\mathrm{es} \& \mathrm{ct}=\mathrm{clnk} \& \mathrm{gl}=\mathrm{mx}$

- Velázquez, E. \& González, I. Reglamento de Zonificación del Estado de Jalisco. Guadalajara, Jal. México: Universidad de Guadalajara, 2003.

- Wolska A. "Human aspects of lighting in working interiors". International Encyclopedia of Ergonomics and Human Factors. Karwowski W. edit. New York: Taylor \& Francis, 2011.

- Yáñez, G. Arquitectura solar e iluminación natural. Madrid, España: Munilla-Lería, 2008. 